\title{
Bumpy Spin-Down of Anomalous X-Ray Pulsars: The Link with Magnetars
}

\author{
A. Melatos \\ Department of Astronomy, 601 Campbell Hall, University of California \\ at Berkeley, Berkeley CA 94720 USA
}

\begin{abstract}
It is argued that bumps in the timing histories $\Omega(t)$ of the anomalous $X$-ray pulsars (AXPs) 1E 1048.1-5937 and 1E 2259+586 are the signature of a magnetar undergoing radiative precession, wherein the hydromagnetic deformation of the neutron star couples to an oscillating component of the vacuum-dipole radiation torque to produce an anharmonic wobble with period $\tau_{\mathrm{pr}} \sim 10 \mathrm{yr}$. An analysis of Euler's equations of motion for a biaxial magnet reproduces the amplitude and recurrence time of the bumps for $1 \mathrm{E} 1048.1-5937$ and $1 \mathrm{E} 2259+586$, predicts $\Omega(t)$ for the next 20 years for both objects, and predicts a testable statistical relation between $d \Omega / d t$ and $\tau_{\text {pr }}$ for the AXP population overall. Radiative precession of soft gamma-ray repeaters is also discussed, together with implications for the internal (e.g. viscosity) and magnetospheric (e.g. $e^{+} e^{-}$pair currents) properties of magnetars.
\end{abstract}

\section{Introduction}

Anomalous X-ray pulsars (AXPs) are a subclass of X-ray pulsars, with pulse periods between 6 and $12 \mathrm{~s}$, for which optical counterparts and orbital Doppler shifts of pulse arrival times have not been detected (Mereghetti \& Stella 1995; van Paradijs, Taam \& van den Heuvel 1995). At present, there is debate over whether AXPs are (i) ordinary neutron stars with surface magnetic field $B_{0} \sim 10^{12} \mathrm{G}$, accreting from a very-low-mass binary companion or circumstellar disk (van Paradijs et al. 1995; Baykal \& Swank 1996), or (ii) magnetars, i.e. nonaccreting, ultramagnetized neutron stars with $B_{0} \gtrsim 10^{14} \mathrm{G}$, spinning down electromagnetically (Thompson \& Duncan 1996; Heyl \& Hernquist 1999).

Two AXPs, $1 \mathrm{E} 1048.1-5937$ and $1 \mathrm{E} 2259+586$, possess well-sampled timing histories extending back over 20 years. Both objects spin down irregularly: the rotation frequency decreases linearly with $t$ on average, but there are 'bumps' superposed on the average trend every $5-10$ yr during which $\dot{\Omega}=d \Omega / d t<0$ fluctuates by a factor of 2-5 (Baykal et al. 1998; Oosterbroek et al. 1998; and references therein). In existing models of AXPs, the bumps are ascribed to white accretion-torque noise (Baykal \& Swank 1996) or Vela-like glitches (Heyl \& Hernquist 1999). Here we discuss an alternative scenario in which the bumps are the spin-down signature of radiative precession of a magnetar (Melatos 1999). 


\section{Radiative Precession}

Internal hydromagnetic stresses deform a magnetar, producing a fractional difference $\epsilon=\left(I_{3}-I_{1}\right) / I_{1} \approx B_{\text {in }}^{2} R^{5} / 4 \pi c_{\mathrm{s}}^{2} I_{1} \approx 2 \times 10^{-9}\left(B_{\text {in }} / 10^{14} \mathrm{G}\right)^{2}$ between the principal moments of inertia $I_{1}$ and $I_{3}$, where $B_{\text {in }}$ is the characteristic strength of the internal magnetic field, $c_{\mathrm{s}} \approx 3^{-1 / 2} c$ is the isothermal sound speed, and $R$ is the stellar radius (Goldreich 1970; Katz 1989; Melatos 1999; see also Jones 1975 for a discussion of how the distortion is amplified by the proton superfluid in the star). One has $B_{\text {in }} \approx B_{0}$ if the internal field is confined within the crust and $B_{\text {in }} \gtrsim B_{0}$ if it is generated in the core; moreover, the principal axis $\mathbf{e}_{3}$ is approximately parallel to $m$, the axis of the external magnetic dipole, provided the source dynamo operates in a roughly axisymmetric, low-order-multipole mode.

In the absence of an external torque, and if $\boldsymbol{\Omega}$ is not parallel to $\mathbf{e}_{3}$, the star precesses freely with period $\tau_{\mathrm{pr}}=2 \pi / \epsilon \Omega \approx 85\left(B_{\mathrm{in}} / 10^{14} \mathrm{G}\right)^{-2}\left(\Omega / 1 \mathrm{rad} \mathrm{s}^{-1}\right)^{-1} \mathrm{yr}$. In reality, an external torque is exerted by the vacuum radiation fields of the rotating magnetic dipole. It consists of two parts: (i) the familiar spin-down torque $\propto \Omega^{3}$, which acts along $\Omega \times(\Omega \times \mathbf{m})$ on the braking time-scale $\tau_{0}=$ $2 c^{3} I_{1} / B_{0}^{2} R^{6} \Omega^{2} \gtrsim 2 \times 10^{5}\left(B_{0} / 10^{14} \mathrm{G}\right)^{-2}\left(\Omega / 1 \mathrm{rad} \mathrm{s}^{-1}\right)^{-2} \mathrm{yr}$, and (ii) a near-field torque $\propto \Omega^{2}$, associated with the axisymmetric inertia of the near-zone radiation fields, which acts along $\Omega \times \mathbf{m}$ on the time-scale $\tau_{\mathrm{nf}} \approx \tau_{0} \Omega R / c \approx$ $6\left(B_{0} / 10^{14} \mathrm{G}\right)^{-2}\left(\Omega / 1 \mathrm{rad} \mathrm{s}^{-1}\right)^{-1} \mathrm{yr}$ (Goldreich $1970 ;$ Melatos 1999$)$. Given $B_{\text {in }} \chi$ $B_{0}$, one finds $\tau_{\mathrm{pr}} \sim \tau_{\mathrm{nf}}$, i.e. the near-field torque couples to the Eulerian precession. The star wobbles anharmonically, with the angle $\alpha$ between $\Omega$ and $\mathbf{m}$ (and hence $\dot{\Omega} \propto \sin ^{2} \alpha$ ) oscillating in a jerky fashion as in Fig. 1a. Each jerk matches a bump in the timing history $\Omega(t){ }^{1}$

Euler's equations of motion for a rotating, biaxial, dipole magnet take the form (Melatos 1999)

$$
\begin{aligned}
\dot{\Omega}_{1}= & -\epsilon \Omega_{2} \Omega_{3}+\Omega_{0}^{-2} \tau_{0}^{-1} \cos \chi\left[a \Omega^{2}\left(-\Omega_{1} \cos \chi+\Omega_{3} \sin \chi\right)\right. \\
& \left.+b \Omega_{2}\left(\Omega_{1} \sin \chi+\Omega_{3} \cos \chi\right)\right], \\
\dot{\Omega}_{2}= & \epsilon \Omega_{1} \Omega_{3}+\Omega_{0}^{-2} \tau_{0}^{-1}\left[-a \Omega^{2} \Omega_{2}\right. \\
& \left.+b\left(-\Omega_{1} \cos \chi+\Omega_{3} \sin \chi\right)\left(\Omega_{1} \sin \chi+\Omega_{3} \cos \chi\right)\right], \\
\dot{\Omega}_{3}= & -\Omega_{0}^{-2} \tau_{0}^{-1} \sin \chi\left[a \Omega^{2}\left(-\Omega_{1} \cos \chi+\Omega_{3} \sin \chi\right)\right. \\
& \left.+b \Omega_{2}\left(\Omega_{1} \sin \chi+\Omega_{3} \cos \chi\right)\right] .
\end{aligned}
$$

Subscripts denote vector components along the principal axes of inertia, $\chi$ is the (fixed) angle between $\mathrm{m}$ and $\mathrm{e}_{3}$, and we have $a=0.33, b=0.094 c / \Omega_{0} R$, and $\Omega_{0}=\Omega\left(t_{0}\right)$, where $t_{0}$ is an arbitrary origin. Terms $\propto \epsilon$ produce Eulerian precession, terms $\propto b$ arise from the near-field torque, and terms $\propto a$ produce secular braking. Equations (1)-(3) can be generalized to accommodate triaxiality (see also Fig. 1a), and one can model crudely the internal magnetization (e.g. toroidal versus poloidal) and distribution of magnetospheric currents (e.g. plasma modifications) by adjusting the values of $a$ and $b$ respectively.

\footnotetext{
${ }^{1}$ The near-field torque, although directed along $\Omega \times \mathbf{m}$, does change $|\Omega|$ because the angular momentum vector is not parallel to $\boldsymbol{\Omega}$ for an aspherical star.
} 

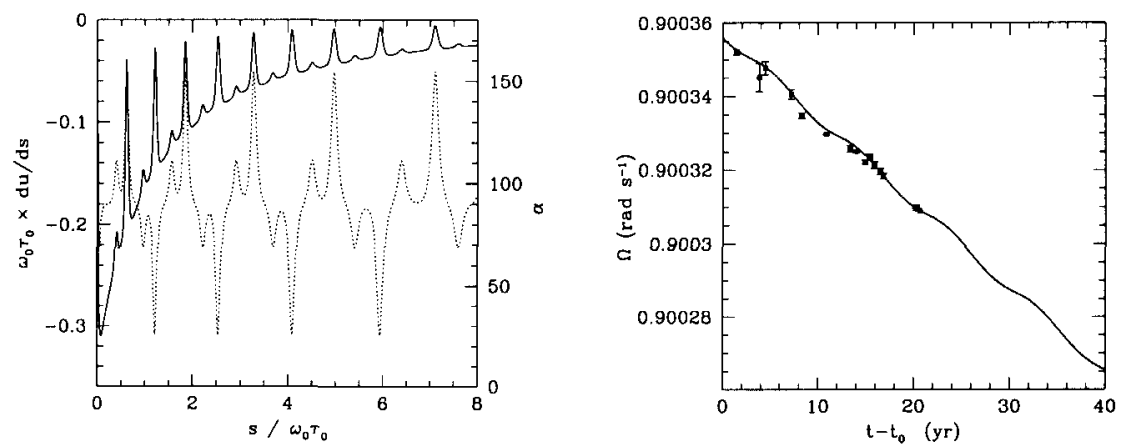

Figure 1. (a) Angular frequency derivative $\dot{\Omega}$ (solid curve), in dimensionless units, and the angle $\alpha$ between $\Omega$ and $\mathbf{m}$ (dotted curve), in degrees, as functions of time, in units of the braking time-scale $\tau_{0}$. Both curves are for $\chi=40^{\circ}, \epsilon=93\left(\Omega_{0} \tau_{0}\right)^{-1}, \epsilon^{\prime}=\left(I_{2}-I_{1}\right) / I_{1}=0.09 \epsilon$, $\Omega_{2,0}=0.28 \Omega_{0}$, and $\Omega_{3,0}=0.40 \Omega_{0}$. A similar effect, without the small secondary bumps, occurs for a biaxial star with $\epsilon^{\prime}=0$. (b) Rotation frequency $\Omega$ vs. time $t$ for the AXP $1 \mathrm{E} 2259+586$, with $t_{0}=\mathrm{JD} 2,443,000$. The squares and $1 \sigma$ error bars are $\mathrm{X}$-ray timing data (Baykal et al. 1998 and references therein). The solid curve is the solution to Euler's equations of motion (eqs. [1]-[3]) for $\Omega_{0}=0.900356 \mathrm{rads}^{-1}$, $\Omega_{0} \tau_{0}=2.35 \times 10^{12}, \epsilon=3.4 \times 10^{-8}$, and $\chi=13^{\circ}$, with initial conditions $\Omega_{2,0}=-0.668 \Omega_{0}$ and $\Omega_{3,0}=0.658 \Omega_{0}$.

\section{X-Ray Timing and Population Statistics}

Fig. 1b displays X-ray-timing data for $1 \mathrm{E} 2259+586$ together with the best theoretical fit from (1)-(3). The unknown parameters $\epsilon, \tau_{0}$ and $\chi$ are constrained to better than 5 per cent, and their values are exactly what one expects if AXPs are hydromagnetically deformed magnetars with $B_{\text {in }} \gtrsim B_{0} \gtrsim 3 \times 10^{14} \mathrm{G}$ and $\chi$ relatively small as for the geodynamo (see $\S 2$ ). A similar conclusion pertains to 1E 1048.1-5937 (Melatos 1999). The future timing behavior predicted by the theory is also tightly constrained - and hence falsifiable. Although a formal estimate of the chi-square of the fit compares unfavorably with alternative models invoking multiple glitches (Heyl \& Hernquist 1999), the $\chi^{2}$ likelihood improves dramatically when triaxiality is added to (1)-(3) and $a$ and $b$ are treated as free parameters (see $\S 2$ ). A detailed comparison of this more general model with available data is in progress.

If radiative precession is responsible for bumpy spin-down, one expects an inverse correlation across the AXP population between bump recurrence time $\tau_{\mathrm{pr}} \propto B_{\mathrm{in}}^{-2}$ and average spin-down rate $\langle\dot{\Omega}\rangle \approx \Omega / \tau_{0} \propto B_{0}^{2}$, viz.

$$
\langle\dot{\Omega}\rangle \approx-2 \times 10^{-4}\left(B_{0} / B_{\mathrm{in}}\right)^{2}\left(\Omega / 1 \mathrm{rad} \mathrm{s}^{-1}\right)^{2}\left(\tau_{\mathrm{pr}} / 1 \mathrm{yr}\right)^{-1} \mathrm{rad} \mathrm{s}^{-1} \mathrm{yr}^{-1},
$$

with some scatter because $\tau_{\mathrm{pr}}$ and $\langle\dot{\Omega}\rangle$ depend on the detailed internal and external magnetizations of each object (Melatos 1999). One also expects a narrow range of bump amplitudes $\Delta \Omega_{\mathrm{pr}} \approx 5 \times 10^{-5}\left(B_{0} / B_{\mathrm{in}}\right)^{2}\left(\Omega / 1 \mathrm{rad} \mathrm{s}^{-1}\right)^{2} \mathrm{rad} \mathrm{s}^{-1}$ (Melatos 1999). 


\section{Internal and Magnetospheric Structure of a Magnetar}

Bumpy spin-down is not observed in rotation-powered pulsars with $B_{0} \lesssim 10^{13} \mathrm{G}$, except for PSR B1828-11 (A. Lyne, this symposium). This may be because radiative precession is viscously damped inside an ordinary pulsar (cf. the Earth), whereas the stiffening action of the superstrong magnetic field in a magnetar hinders the development of elastic strains and sheared fluid flows. Alternatively, it may imply that conduction currents in the magnetosphere of an ordinary pulsar nullify the precessive near-field torque, whereas the vacuum fields and hence the near-field torque are not modified in a magnetar because pair production is quenched, e.g. by positronium formation.

Soft gamma-ray repeaters (SGRs) are also thought to be magnetars. Woods et al. (1999) recently presented Rossi X-Ray Timing Explorer observations of SGR $1900+14$ that reveal bumpy spin-down in that object, with $\dot{\Omega}$ changing by a factor $\approx 2.3$ during an interval of $\approx 80 \mathrm{~d}$ before reverting to the trend rate $\langle\dot{\Omega}\rangle$. The data are consistent with radiative precession. However, the $80-\mathrm{d}$ interval coincided with a giant X-ray flare which initiated several months of burst activity. A correlation between flares and bumpy spin-down is not expected in the simplest radiative-precession scenario. If future data substantiate such a correlation, an alternative picture becomes more likely in which Eulerian precession and/or Vela-like glitches are excited by some flare trigger, such as episodic Alfvén-wave emission or a starquake (Heyl \& Hernquist 1999; Woods et al. 1999; C. Thompson, this symposium).

Acknowledgments. This work was supported by NASA Grant NAG53073, NSF Grant AST-95-28271, and by the Miller Institute for Basic Research in Science through a Miller Fellowship.

\section{References}

Baykal, A., \& Swank, J. 1996, ApJ, 460, 470

Baykal, A., Swank, J. H., Strohmayer, T., \& Stark, M. J. 1998, A\&A, 336, 173

Goldreich, P. 1970, ApJ, 160, L11

Heyl, J. S., \& Hernquist, L. 1999, MNRAS, 304, L37

Jones, P. B. 1975, Ap\&SS, 33, 215

Katz, J. I. 1989, MNRAS, 239, 751

Melatos, A. 1999, ApJ, 519, L77

Mereghetti, S., \& Stella, L. 1995, ApJ, 442, L17

Oosterbroek, T., Parmar, A., Mereghetti, S., \& Israel, G. 1998, A\&A, 334, 925

Thompson, C., \& Duncan, R. C. 1996, ApJ, 473, 322

van Paradijs, J., Taam, R. E., \& van den Heuvel, E. P. J. 1995, A\&A, 299, L41

Woods, P. M., et al. 1999, ApJ, 524, L55 